\title{
Evolución de la capacidad de cambio de dirección en función de la edad en niños y niñas en edad escolar
}

\section{Evolution of the change of direction ability according to the age in school-age children}

\author{
Josune Rodríguez-Negro*, Estibaliz Romaratezabala y Javier Yanci
}

Departamento de Educación Física y Deportiva, Facultad de Educación y Deporte. Universidad del País Vasco, UPVIEHU, Vitoria-Gasteiz, España.

\begin{abstract}
Resumen: Los objetivos de este estudio fueron describir la capacidad de cambio de dirección (CODA) en estudiantes de educación primaria (EP, $\mathrm{n}$ = 394) y analizar las diferencias atendiendo a su edad. Se han encontrado diferencias significativas en la CODA entre todos los cursos, excepto entre G1-G2 ( $p>0,05$, TE = -0,3, bajo), G1-G3 ( $p>0,05$, TE = -0,3, bajo), G2$\mathrm{G} 3(\mathrm{p}>0,05, \mathrm{TE}=0,1$, trivial), G4-G6 ( $>>0,05, \mathrm{TE}=-0,5$, moderado) y G5-G6 (p > 0,05, TE = 0,1, trivial). En el presente estudio no se han observado diferencias significativas en la CODA entre estudiantes de primero, segundo y tercero de educación primaria (6-8 años), pero si a partir de esa edad (9-12 años). Estas diferencias han sido especialmente relevantes entre G3 y G5. Estos hallazgos nos llevan a pensar que la edad de los 8-9 ańos puede ser una etapa crítica diferenciadora en la CODA de los estudiantes de EP.

Palabras clave: Educación primaria, motricidad, agilidad, MAT, maduración.
\end{abstract}

Abstract: The aims of this study were to describe the change of direction ability (CODA) in primary school students $(n=394)$ and to analyze the differences attending to their age. Significant differences were found in CODA between all courses, except between G1-G2 $(\mathrm{p}>0.05$, ES = 0.3 , small), G1-G3 ( $>>0.05$, ES $=-0.3$, small), G2-G3 ( $>>0.05$, ES $=0.1$, trivial), G4-G6 ( p > 0.05, ES = -0.5, moderate) and G5-G6 (p > $0.05, \mathrm{ES}=0.1$, trivial). In the present study no significant differences were observed in the CODA among first, second and third grade students of primary education (6 to 8 years), but significant differences were observed from that age ( 9 to 12 years). These differences have been especially relevant between G3 and G5. These findings lead us to think that the age of 8-9 years can be a critical differentiating stage in the CODA of the primary education students.

Keywords: Primary education, motor skills, agility, MAT, maturation.

\section{Introducción}

La capacidad de cambio de dirección (CODA) es una habilidad motora compleja (Serpell, Ford y Young, 2010) y un importante componente que interviene en multitud de actividades recreativas, tareas de iniciación deportiva y modalidades deportivas (Sassi, et al., 2009; Sporis, Jukic, Milanovic y Vucetic, 2010), siendo una habilidad necesaria para que los niños y niñas alcancen un adecuado desempeño motor. Por esta razón son varios los estudios que han evaluado la CODA en niños y niñas en educación física (Mckenzie, et al., 2002; Yanci, et al., 2015) o en iniciación deportiva (Meylan, Cronin, Oliver, Hopkins y Pinder, 2014; Yanci, Cámara, Vizcay y Young, 2016).

Para la evaluación de la CODA se han utilizado diferentes test como el Star Agility Run Test (Golle, Muehlbauer, Wick y Granacher, 2015; Granacher y Borde, 2017), el Illinois Agility Test (Ozmen y Aydogmus, 2016; Raya et al., 2013) o el Modified Illinois Change ofDirection Test (Hachanaet al., 2014; Negra et al., 2016) entre otros. Sin embargo, en el

Dirección para correspondencia [Correspodence address]: Josune Rodríguez-Negro. Departamento de Educación Física y Deportiva, Facultad de Educación y Deporte. Universidad del País Vasco, UPV/ EHU, Vitoria-Gasteiz (Espańa). E-mail: jrodriguez168@ehu.eus contexto escolar, uno de los más utilizados es el Modified Agility Test (MAT), que ha demostrado buenos valores de reproducibilidad en estudiantes de educación primaria (EP) (Yanci, Los Arcos, Reina, Gil y Grande, 2014b). La variedad de aspectos que se integran en la CODA hace que su desarrollo sea de gran importancia en las etapas de crecimiento del niño (Yanci et al. 2015), siendo la etapa de EP un momento idóneo para trabajarla. El crecimiento y desarrollo de los niños y niñas es un proceso complejo e interesante (Pikel et al., 2017) que influye en sus capacidades, así como en su CODA (Erceg, Zagorac y Katic, 2008; McArdle, Katch y Katch, 2010). Por esta razón varios estudios han evaluado las diferencias en función de la edad en la CODA en escolares de EP (Meylan, Cronin, Oliver y Rumpf, 2014; Yanci et al., 2014b). Aun así, existe una controversia en la literatura científica sobre el efecto de la edad en la CODA en estudiantes de EP, ya que mientras que algunos autores han encontrado diferencias en la CODA atendiendo a la edad (Erceg, et al., 2008; Yanci, Los Arcos, Grande, Gil y Cámara, 2014a), otros estudios concluyen que la edad y la CODA no están relacionadas (Chatterjee y Bandyopadhyay, 1993). Por lo tanto, son necesarios más estudios que analicen los efectos de la edad en la CODA de estudiantes de EP. 
Por esta razón, los objetivos de este estudio fueron, por un lado, describir la capacidad de cambio de dirección en estudiantes de educación primaria y, por otro lado, analizar las diferencias atendiendo al curso escolar que cursaban los estudiantes.

\section{Material y Método}

\section{Participantes}

En este estudio participaron 394 estudiantes de $1^{\circ}$ a $6^{\circ}$ curso de EP de un colegio público, de los cuales 194 eran chicos $(8,65 \pm 1,85$ años, $1,34 \pm 0,11 \mathrm{~m}, 32,27 \pm 8,38 \mathrm{~kg}, 17,71$ $\left.\pm 2,60 \mathrm{~kg} \cdot \mathrm{m}^{-2}\right)$ y 200 eran chicas $(8,56 \pm 1,64$ años, $1,34 \pm$ $\left.0,11 \mathrm{~m}, 33,43 \pm 10,38 \mathrm{~kg}, 18,20 \pm 3,32 \mathrm{~kg} \cdot \mathrm{m}^{-2}\right)$. El total de la muestra fue dividido en seis grupos atendiendo al curso escolar que cursaban los estudiantes, denominados, primer curso de educación primaria $(\mathrm{G} 1, \mathrm{n}=60)$, segundo curso (G2, $\mathrm{n}=74)$, tercer curso (G3, $\mathrm{n}=62)$, cuarto curso (G4, $\mathrm{n}=64)$, quinto curso $(\mathrm{G} 5, \mathrm{n}=64)$ y sexto curso $(\mathrm{G} 6, \mathrm{n}=70)$. En la tabla 1 se presentan la edad, la masa corporal, la altura y el índice de masa corporal del total de la muestra y de cada uno de los grupos. Antes de comenzar el estudio, todos los participantes fueron informados de los procedimientos, beneficios y posibles riesgos del estudio y todos los padres, madres o tutores legales firmaron el consentimiento informado. El estudio siguió las pautas marcadas en la Declaración de Helsinki (2013) y fue aprobado por el Comité de Ética para la Investigación con Seres Humanos (CEISH: 2015/147) de la Universidad del País Vasco (UPV/EHU).

Tabla 1. Características de los estudiantes.

\begin{tabular}{lllll} 
& Edad(años) & Masa $(\mathrm{kg})$ & Altura $(\mathrm{m})$ & IMC $\left(\mathrm{kg} \cdot \mathrm{m}^{-2)}\right.$ \\
\hline Todos & $8,60 \pm 1,75$ & $32,86 \pm 9,46$ & $1,34 \pm 0,11$ & $17,96 \pm 3,00$ \\
G1 & $6,02 \pm 0,13$ & $24,30 \pm 4,79$ & $1,19 \pm 0,06$ & $16,96 \pm 2,33$ \\
G2 & $7,09 \pm 0,29$ & $27,01 \pm 4,99$ & $1,27 \pm 0,05$ & $16,68 \pm 2,45$ \\
G3 & $8,10 \pm 0,29$ & $31,88 \pm 7,29$ & $1,31 \pm 0,05$ & $18,21 \pm 3,05$ \\
G4 & $9,06 \pm 0,24$ & $34,27 \pm 7,21$ & $1,36 \pm 0,06$ & $18,30 \pm 2,58$ \\
G5 & $10,06 \pm 0,24$ & $37,62 \pm 9,14$ & $1,42 \pm 0,06$ & $18,40 \pm 3,38$ \\
G6 & $11,11 \pm 0,32$ & $41,93 \pm 9,32$ & $1,46 \pm 0,07$ & $19,30 \pm 3,28$ \\
\hline
\end{tabular}

IMC = Índice de masa corporal. G1-6: grupo atendiendo al curso escolar.

\section{Procedimiento}

Al final del primer trimestre escolar (diciembre), se evaluó la CODA de los estudiantes durante una sesión de EF. El test se realizó en horario de mañana, en el polideportivo en el que los estudiantes realizaban habitualmente las sesiones de EF.

Modified Agility Test (MAT):Para evaluar la CODA se re- alizó el MAT siguiendo el protocolo establecido por Yanci, Reina, Los Arcos y Cámara (2013)para estudiantes de EP. La distancia total recorrida fue de $20 \mathrm{~m}$ y para medir el tiempo empleado en realizar el recorrido se colocaron unas fotocélulas (Microgate ${ }^{\mathrm{TM}}$ Polifemo Radio Light, Bolzano, Italy) en la línea de inicio/final. En la figura 1 se presenta el recorrido realizado por los estudiantes para completar el test. Todos los participantes realizaron el test 3 veces con un descanso de 2 min entre cada intento. El mejor de los tres intentos fue elegido para el análisis estadístico. Todos los alumnos estaban familiarizados con la ejecución correcta del test porque en las sesiones previas recibieron explicaciones y pudieron practicarlo.

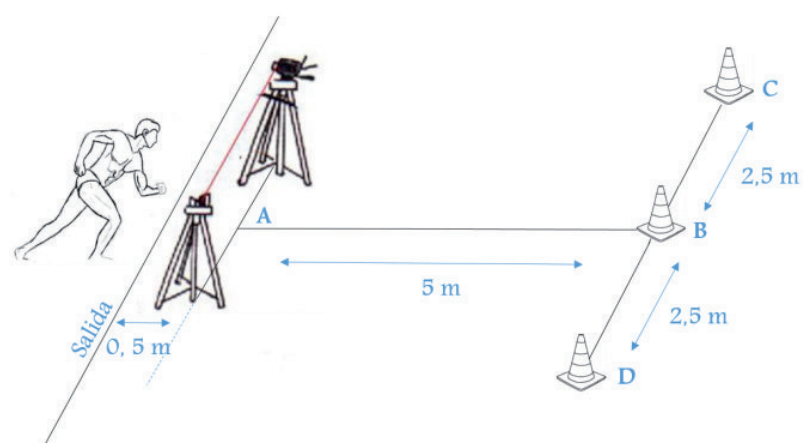

Figura 1. Recorrido realizado por los participantes en el Modified Agility Test (MAT).

\section{Análisis estadístico}

Los resultados se presentan como media \pm desviación típica (DT) de la media.Para analizar las diferencias entre los resultados del MAT para los grupos de edad (G1-G6) se utilizó un ANOVA de un factor con el post hoc de Bonferroni.La diferencia en porcentaje $(\Delta \%)$ se calculó mediante la fórmula: $\Delta(\%)=[($ media $2-$ media 1$) /$ media 1$] \times 100$ en cada uno de los posibles casos. Para determinar las diferencias a efectos prácticos se utilizó el tamaño del efecto (TE) propuesto por Cohen (1988). Tamańos del efecto menores a 0,2, entre 0,2-0,5, entre 0,5-0,8 y mayores de 0,8 fueron considerados trivial, bajo, moderado y alto, respectivamente. El análisis estadístico se realizó con el programa StatisticalPackagefor Social Sciences (SPSS Inc, versión 22,0 Chicago, IL, EE.UU.). La significatividad estadística fue de $\mathrm{p}<0,05$.

\section{Resultados}

El resultado obtenido por todos los estudiantes en el MAT fue de 10,35 $\pm 1,69$ s. En la Figura 1 se presentan los resultados descriptivos del MAT para cada grupo de edad. 


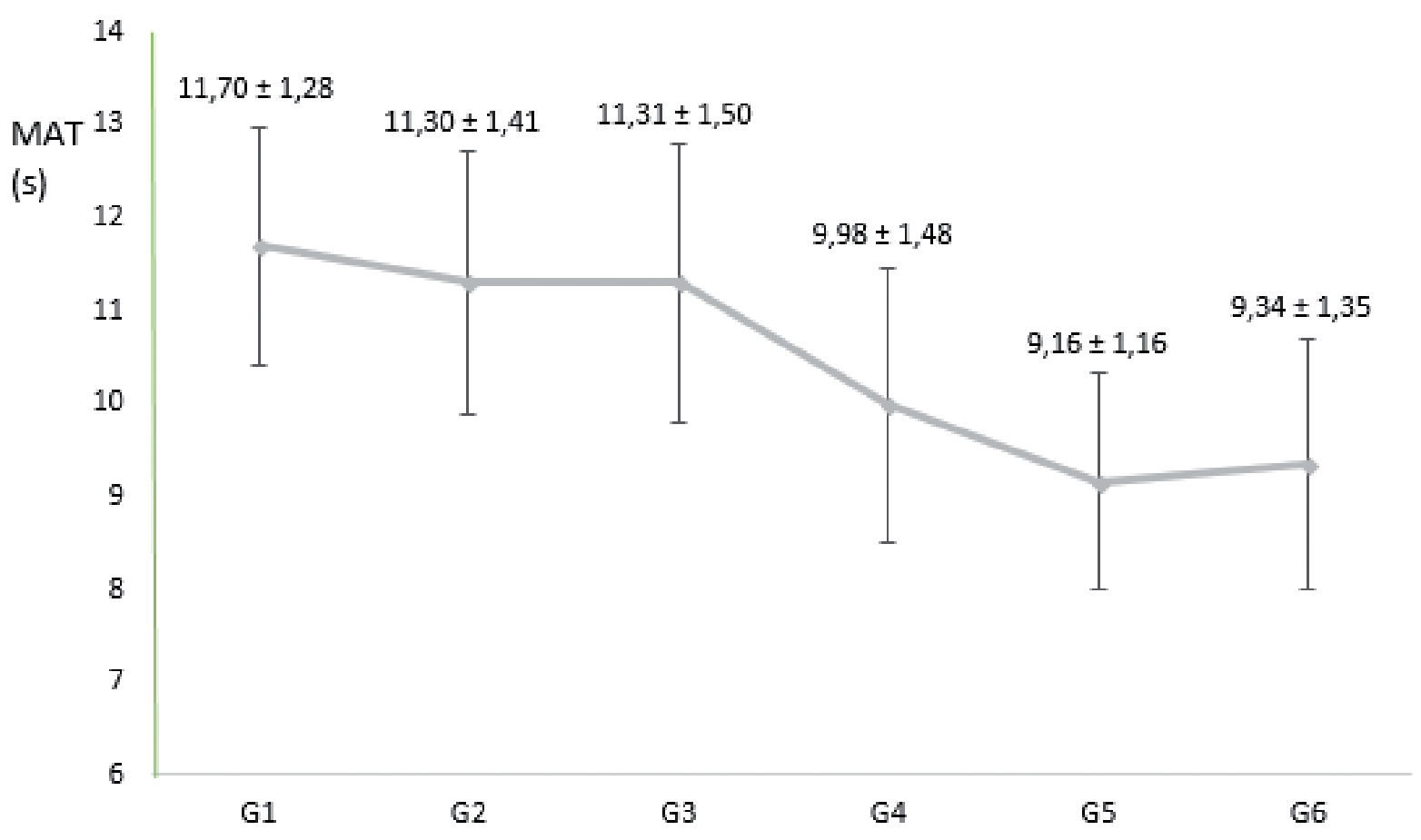

Figura 1. Resultados del Modified Agility Test (MAT) para cada grupo de edad.

G1-6 = Curso de educación primaria.

En la tabla 2 se presentan las diferencias en los resultados del MAT entre los diferentes grupos de edad. Se encontraron diferencias significativas en el MAT entre todos los cursos, excepto entre G1-G2 ( $p>0,05, \mathrm{TE}=-0,3$, bajo), G1-G3 ( $p$
$>0,05, \mathrm{TE}=-0,3$, bajo), G2-G3 $(\mathrm{p}>0,05, \mathrm{TE}=0,1$, trivial $)$, G4-G6 (p > 0,05, TE = -0,5, moderado) y G5-G6 ( p > 0,05, $\mathrm{TE}=0,1$, trivial).

Tabla 2. Diferencias en los resultados del MAT entre los diferentes grupos de edad.

\begin{tabular}{llllllllllllllll}
\hline & G1-G2 & G1-G3 & G1-G4 & G1-G5 & G1-G6 & G2-G3 & G2-G4 & G2-G5 & G2-G6 & G3-G4 & G3-G5 & G3-G6 & G4-G5 & G4-G6 & G5-G6 \\
\hline$\Delta \%$ & $-3,4$ & $-3,3$ & $-14,7$ & $-21,7$ & $-20,2$ & 0,1 & $-11,7$ & $-18,9$ & $-17,3$ & $-11,7$ & $-19,0$ & $-17,4$ & $-8,2$ & $-6,2$ & 2,0 \\
TE & $-0,3$ & $-0,3$ & $-1,2^{* *}$ & $-2,2^{* *}$ & $-1,7^{* *}$ & 0,1 & $-0,9^{* *}$ & $-1,8^{* *}$ & $-1,4^{* *}$ & $-0,9^{* *}$ & $-1,8^{* *}$ & $-1,4^{* *}$ & $-0,7^{*}$ & $-0,5$ & 0,1 \\
\hline
\end{tabular}

$\Delta \%=$ Diferencia en porcentaje, TE $=$ Tamaño del efecto, G1-6 = grupo atendiendo al curso. Diferencias significativas $\left({ }^{*} \mathrm{p}<0,05,{ }^{* *} \mathrm{p}<0,01\right)$.

\section{Discusión}

La principal contribución del presente estudio ha sido la descripción de la CODA, así como el análisis de las diferencias en la CODA atendiendo al curso escolar en estudiantes de EP (G1-G6). Los resultados obtenidos en este estudio muestran un tiempo de 10,35 $\pm 1,69 \mathrm{~s}$ en la realización del test MAT para todos los estudiantes. Estos resultados son similares a los obtenidos por Yanci et al. (2014b) también en estudiantes de EP. Sin embargo, son mayores a los obtenidos por jóvenes atletas de una escuela de atletismo (Yanci, Los Arcos, Castillo y Cámara, In press), con una diferencia de entre un 24,9\% y un $30,9 \%$ dependiendo de la edad. Algunos estudios han analizado la influencia de la práctica de una modalidad deportiva concreta en las habilidades motoras (Sekulic, Spasic,
Mirkov, Cavar y Sattler, 2013) y exponen que el hecho de practicar algunas modalidades deportivas puede mejorar la CODA en practicantes en edad de iniciación (Katis y Kellis, 2009; Oxyzoglou, Kanioglou y Ore, 2009). Concretamente, Yanci, Vizcay, Pitillas y Los Arcos (2016), analizaron las diferencias en la CODA entre estudiantes de EP y atletas de la misma edad de una escuela de atletismo, y obtuvieron que los atletas tenían una mejor CODA que los estudiantes que no realizaban una actividad deportiva reglada fuera del horario escolar.

Por otra parte, los resultados obtenidos en el presente estudio no mostraron diferencias significativas en la CODA entre los estudiantes de primero, segundo y tercero de EP (6 a 8 años). Sin embargo, estas diferencias si fueron significativas a partir de los 9 años (G4 en adelante, 9 a 12 años). Singh, 
Joon y Kooner (1987) analizaron la CODA en niños de entre 9 y 16 años y al igual que en nuestro estudio, concluyeron que a partir de los 9 años la edad de los estudiantes influía en su CODA.En nuestro estudio se encontraron diferencias en la CODA entre grupos de edad a partir de los 9 ańos, reforzando la hipótesis de que la edad puede ser un factor diferenciador en las habilidades de los estudiantes de EP, concretamente en la CODA. Estos resultados coinciden parcialmente con los resultados obtenidos en otros estudios donde también se ha observado una mejora en las habilidades motrices básicas y la coordinación motora gruesa durante el desarrollo infantil (Bigoniet al., 2017; Steindl, Kunz, Schrott-Fischer y Scholtz, 2006). A pesar de que los resultados de la presente investigación muestran que los estudiantes de EP de mayor edad obtienen mejor CODA que los estudiantes de cursos inferiores y que especialmente se produce una mayor mejora entre los cursos G3 y G5, no se han obtenido diferencias en la CODA en los primeros cursos de EP (G1-G3). Posiblemente, a partir de los 8-9 años la maduración, el aumento de la masa muscular, y el aumento de la capacidad de generar fuerza, pueden influir en el rendimiento de CODA (Castro-Piñeiro et al., 2010; Tercedor, et al., 2003). Además, a esa edad (8-9 años) comienza una fase transitoria y crítica para la adquisición de habilidades motoras (Gallahue, Ozmun, Araújo, de Medeiros Ribeiro y Silva, 2005), y se produce un mayor aumento de las actividades pre deportivas o deportivas (CSD, 2015) y por tanto quizá al tener los estudiantes más estímulos que en edades anteriores se da una mejora relevante. Por esta razón, los docentes de EF durante la etapa de EP deberían de tener en cuenta la edad de los estudiantes, porque esta puede influenciar en la CODA. A pesar de que durante la etapa de EP se dan mejoras significativas en la CODA de los estudiantes, posiblemente a partir de los 8-9 ańos sea un momento clave para su mejora.

\section{Conclusiones}

En el presente estudio no se han observado diferencias significativas en la CODA entre estudiantes de primero, segundo y tercero de educación primaria (6 a 8 años), pero si a partir de esa edad ( 9 a 12 años). Estas diferencias han sido especialmente relevantes entre G3 y G5. Estos hallazgos nos llevan a pensar que la edad de los 8-9 años puede ser una etapa crítica diferenciadora en la CODA de los estudiantes de EP. Por esta razón, resulta interesante que los docentes de EF durante la etapa de EP tengan en cuenta las mejoras en la CODA según la edad a la hora de diseñar los ejercicios de las sesiones de EF, aumentando su dificultad e intensidad a medida que los estudiantes crecen.

\section{Agradecimientos}

Este trabajo fue financiado por el Gobierno Vasco a través de una beca predoctoral cuyo número de referencia es PRE_2016_1_0171.

\section{Referencias bibliográficas}

1. Bigoni, M., Turati, M., Gandolla, M., Augusti, C. A., Pedrocchi, A., La Torre, A., ... \&Gaddi, D. (2017). Balance in young male soccer players: dominant versus non-dominant leg. Sport Sciences for Health, 13(2), 253-258.Doi: 10.1007/s11332-016-0319-4.

2. Castro-Piñero, J., González-Montesinos, J.L., Keating, X.D., Mora, J., Sjöström, M.,\& Ruiz, J.R. (2010). Percentile values for running sprint field tests in children ages 6-17 years: influence of weight status. Research Quarterly in Exercise and Sport, 81(2), 143-151. Doi: 10.1080/02701367.2010.10599661.

3. Chatterjee, S.,\& Bandyopadhyay, A. (1993). Effect of continuous slowspeed running for 12 weeks on 10-14-year-old Indian boys. British Journal of Sports Medicine, 27(3), 179-185.Doi: 10.1136/bjsm.27.3.179.

4. Cohen, J. (1988). Statistical power analysis for the behavioural sciences. New York: AcademicPress.

5. Consejo Superior de Deportes. (2015). Los hábitos deportivos de la población escolar en España. Madrid: Consejo Superior de Deportes.

6. Erceg, M., Zagorac, N., \&Katic, R. (2008). Theimpactoffootball training on motor development in male children. CollegiumAntropologicum, 32(1), 241-247.

7. Gallahue, D. L., Ozmun, J. C., Araújo, M. A. S. P., de Medeiros Ribeiro, J.,\& Silva, J. P. S. (2005). Compreendendo o desenvolvimento motor: bebês, crianças, adolescentes e adultos. São Paulo: Phorte.

8. Golle, K., Muehlbauer, T., Wick, D.,\&Granacher, U. (2015). Physical Fitness Percentiles of German Children Aged 9-12 Years: Findings from a Longitudinal Study. PLoS ONE, 10(11).Doi:10.1371/journal. pone. 0142393.

9. Granacher, U.,\&Borde, R. (2017). Effects of Sport-Specific Training during the Early Stages of Long-Term Athlete Development on Physical Fitness, Body Composition, Cognitive, and Academic Performances. Frontiers in Physiology, 8, 810.Doi: 10.3389/fphys.2017.00810.

10. Hachana, Y., Chaabene, H., Ben Rajeb, G., Khlifa, R., Aouadi, R., Chamari, K., ... \& \&abbett, T. J. (2014). Validity and reliability of new agility test among elite and subelite under 14-soccer players. PLoS One, 9, e95773.Doi: 10.1371/journal.pone.0095773.

11. Katis, A., \&Kellis, E. (2009). Effects of small-sided games on physical conditioning and performance in young soccer players. Journal of Sports Science and Medicine, 8(3), 374-380.

12. McArdle, W. D., Katch, F. I.,\&Katch, V. L. (2010). ExercisePhysiology: Nutrition, Energy, and Human Performance (7th ed.). Philadelphia: Lippincott Williams and Wilkins.

13. McKenzie, T. L., Sallis, J. F., Broyles, S. L., Zive, M. M., Nader, P. R., Berry, C. C., ... \&Brennan, J. J. (2002). Childhood Movement Skills: Predictors of Physical Activity in Anglo American and Mexican American Adolescents? Research Quarterly for Exercise and Sport, 73(3), 238244.Doi: 10.1080/02701367.2002.10609017.

14. Meylan, C. M., Cronin, J. B., Oliver, J. L., Hopkins, W. G.,\& Pinder, S. (2014). Contribution of vertical strength and power to sprint performance in young male athletes. International Journal of Sports Medicine, 35(9), 749-754. Doi: 10.1055/s-0033-1363191. 
15. Meylan, C. M., Cronin, J. B., Oliver, J. L.,\& Rumpf, M. C. (2014). Sexrelated differences in explosive actions during late childhood. Journal of Strength and Conditioning Research, 28(8), 2097-2104. Doi: 10.1519/ JSC.0000000000000377.

16. Negra, Y., Chaabene, H. M., Hachana, Y.,\&Granacher, U. (2016). Effects of high-velocity resistance training on athletic performance in prepuberal male soccer athletes. Journal of Strength and Conditioning Research, 30(12), 3290-3297.Doi: 10.1519/JSC.0000000000001433.

17. Oxyzoglou, N., Kanioglou, A.,\& Ore, G. (2009). Velocity, agility, and flexibility performance after handball training versus physical education program for preadolescent children. Perceptual and Motor Skills, 108(3), 873-877.Doi: 10.2466/PMS.108.3.873-877.

18. Ozmen, T.,\& Aydogmus, M. (2016). Effect of core strength training on dynamic balance and agility in adolescent badminton players. Journal of Bodywork and Movement Therapies, 20(3), 565-570. Doi: 10.1016/j. jbmt.2015.12.006.

19. Pikel, T. R., Starc, G., Strel, J., Kovač, M., Babnik, J.,\&Golja, P. (2017). Impactofprematurityon exercise capacity and agility of children and youth aged 8 to 18. Early Human Development, 110, 39-43.Doi: 10.1016/j.earlhumdev.2017.04.015.

20. Raya, M. A., Gailey, R. S., Gaunaurd, I. A., Jayne, D. M., Campbell, S. M., Gagne, E., ... \& \& Tucker, C. (2013). Comparison of three agility tests with male servicemembers: Edgren Side Step Test, T-Test, and Illinois Agility Test. Journal of Rehabilitation Research and Development, 50(7), 951-960. Doi: 10.1682/JRRD.2012.05.0096.

21. Sassi, R. H., Dardouri, W., Yahmed, M. H., Gmada, N., Mahfoudhi, M. E.,\& Gharbi, Z. (2009). Relative and absolute reliability of a modified agility T-test and its relationship with vertical jump and straight sprint. Journal of Strength and Conditioning Research, 23(6), 16441651. Doi: 10.1519/JSC.0b013e3181b425d2.

22. Sekulic, D., Spasic, M., Mirkov, D., Cavar, M.,\& Sattler, T. (2013). Gender-specific influences of balance, speed, and power on agility performance. Journal of Strength and Conditioning Research, 27(3), 802811.Doi: 10.1519/JSC.0b013e31825c2cb0.

23. Serpell, B. G., Ford, M.,\& Young, W. B. (2010). Thedevelopmentof a new test of agility for rugby league. Journal of Strength and Conditioning Research, 24(12), 3270-3277. Doi: 10.1519/JSC.0b013e3181b60430.
24. Singh, H., Joon, D. S.,\& Kooner, K. (1987). Development of motor abilities of trained Indian boys of 9-16 years of age. British Journal ofSports Medicine, 21(2):34-35.

25. Sporis, G., Jukic, I., Milanovic, L.,\& Vucetic, V. (2010). Reliability and factorial validity of agility tests for soccer players. Journal of Strength and Conditioning Research, 24(3), 679-686.Doi: 10.1519/ JSC.0b013e3181c4d324.

26. Steindl, R., Kunz, K., Schrott-Fischer, A.,\& Scholtz, A. W. (2006). Effect of age and sex on maturation of sensory systems and balance control. Developmental Medicine and Child Neurology, 48(6), 477-482. Doi: 10.1017/S0012162206001022.

27. Tercedor, P., Gonzalez-Gross, M., Delgado, M., Chillón, P., Pérez, I.,\& Ruiz, J. R. (2003). Motives and frequency of physical activity practiced in Spanish adolescents. The AVENA study. Annals of Nutrition \&Metabolism, 47, 499

28. Yanci, J., Cámara, J., Vizcay, J.,\& Young, B. W. (2016). Examining age and gender effects in physical performance in young athletes aged 12-16 years. International Journal of Sports Science \& Coaching, 11(4), 538-544.Doi: 10.1177/1747954116655052.

29. Yanci, J., Los Arcos, A., Castillo, D.,\& Cámara, J. (In press). Sprinting, change of direction ability and horizontal jump performance in youth runners according to gender. Journal of Human Kinetics, 60.

30. Yanci, J., Los Arcos, A., Grande, I., Gil, E.,\&Cámara, J. (2014a). Correlation between agility and sprinting according to student age. CollegiumAntropologicum, 38(2), 533-538.

31. Yanci, J., Los Arcos A., Reina, R., Gil, E.,\& Grande, I. (2014b). La agilidad en alumnos de educación primaria: diferencias por edad y sexo. Revista Internacional de Medicina y Ciencias de la Actividad Física y del Deporte, 53(14), 23-35.

32. Yanci, J., Los Arcos, A., Salinero, J. J., Plana, C., Gil, E., \& Grande, I. (2015). Efectos producidos por diferentes programas de interferencia contextual en la agilidad. Revista Internacional de Medicina y Ciencias de la Actividad Física y el Deporte, 15(59), 405-418.

33. Yanci, J., Reina, R., Los Arcos, A.,\& Cámara, J. (2013). Effects of different contextual interference training programs on straight sprinting and agility performance of primary school students. Journal of Sports Science and Medicine, 12(3), 601-607. 